\title{
MOTION PLANNING FOR A NONLINEAR STEFAN PROBLEM
}

\author{
William B. Dunbar ${ }^{1}$, Nicolas Petit ${ }^{2}$, Pierre Rouchon $^{2}$ and Philippe Martin ${ }^{2}$
}

\begin{abstract}
In this paper we consider a free boundary problem for a nonlinear parabolic partial differential equation. In particular, we are concerned with the inverse problem, which means we know the behavior of the free boundary a priori and would like a solution, e.g. a convergent series, in order to determine what the trajectories of the system should be for steady-state to steady-state boundary control. In this paper we combine two issues: the free boundary (Stefan) problem with a quadratic nonlinearity. We prove convergence of a series solution and give a detailed parametric study on the series radius of convergence. Moreover, we prove that the parametrization can indeed can be used for motion planning purposes; computation of the open loop motion planning is straightforward. Simulation results are given and we prove some important properties about the solution. Namely, a weak maximum principle is derived for the dynamics, stating that the maximum is on the boundary. Also, we prove asymptotic positiveness of the solution, a physical requirement over the entire domain, as the transient time from one steady-state to another gets large.
\end{abstract}

Mathematics Subject Classification. 93C20, 80A22, 80A23.

Received October 31, 2002. Revised January 24, 2003.

\section{INTRODUCTION}

In this paper we consider a free boundary problem for a nonlinear parabolic partial differential equation. In particular, we are concerned with the inverse problem, which means we know the behavior of the free boundary a priori and would like a solution, e.g. a convergent series, in order to determine what the trajectories of the system should be for steady-state to steady-state boundary control.

The classical Stefan problem models a column of liquid in contact at 0 degrees with an infinite strip of ice, as depicted in Figure 1. The problem is thoroughly explored in [1] and a catalogue of various problems reducing to problems of the Stefan type is given in [14]. We investigate a modified Stefan problem that includes a diffusion term and a nonlinear reaction term. This can be seen as a simple model of a chemically reactive and heat diffusive liquid surrounded by ice, as considered under a more general form in [2].

Define $(x, t) \mapsto u(x, t)$ as the temperature in the liquid and $t \mapsto y(t)$ as the position of the liquid/solid interface, given a position $x$ and time $t$. The functions $h(t)$ and $\psi(x)$ are the temperatures at the fixed end $(x=0)$ and at initial time $(t=0)$, respectively. The nonlinear Stefan problem is to determine a $u(x, t)$ and $y(t)$,

Keywords and phrases: Inverse Stefan problem, flatness, motion planning.

1 Control and Dynamical Systems, California Institute of Technology, Mail Code 107-8l, 1200 E California Blvd., Pasadena, CA 91125, USA.

2 Centre Automatique et Systèmes, École Nationale Supérieure des Mines de Paris, 60 boulevard Saint-Michel, 75272 Paris Cedex 06, France; e-mail: petit@cas.ensmp.fr 


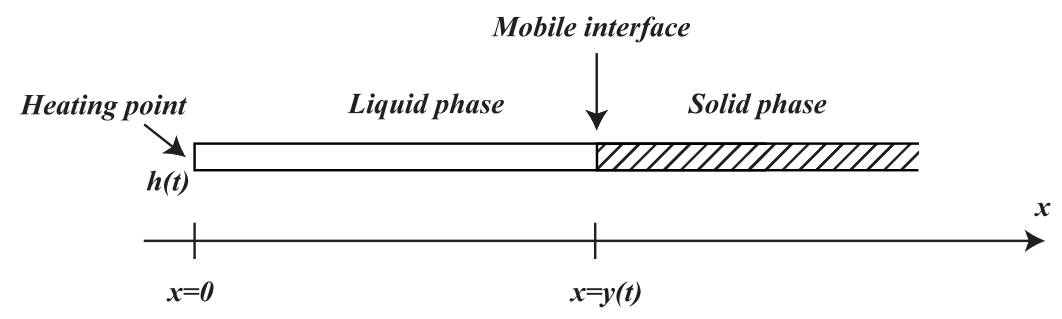

FiguRE 1. The system under consideration: liquid phase governed by a nonlinear heat equation with boundary control, in contact with an infinite solid phase.

given $h(t)$ and $\psi(x)$, that satisfy

$$
\left.\begin{array}{ll}
u_{t}=u_{x x}-\nu u_{x}-\rho u^{2}, & \forall(x, t) \in D_{T}, \\
u(0, t)=h(t) \geq 0, & 0<t \leq T \\
u(x, 0)=\psi(x) \geq 0, & 0 \leq x \leq y(0) \\
u(y(t), t)=0, u_{x}(y(t), t)=-\dot{y}(t), & 0<t \leq T
\end{array}\right\}
$$

where

$$
D_{T} \equiv\{(x, t): 0<x<y(t), 0<t \leq T\},
$$

and the boundaries defined in the last three conditions are denoted in order as

$$
B_{T} \equiv\{(0, t): 0<t \leq T\} \cup\{(x, 0): 0 \leq x \leq y(0)\} \cup\{(y(t), t): 0<t \leq T\} \equiv B_{T}^{1} \cup B_{T}^{2} \cup B_{T}^{3} .
$$

The notation $\dot{y}(t)$ is the time derivative of $y(t)$ and $\nu, \rho \geq 0, T>0$. This model arises from a classical energy balance. The equation $u_{x}(y(t), t)=-\dot{y}(t)$ expresses the fact that all of the heat energy arriving at the liquid-solid interface is utilized in the melting process. In the model, the thermal conductivity coefficient and a parameter equal to the product of the solid density and the latent heat of fusion are normalized to one. Of course it is possible to use different values for these coefficients using changes of scales for $x, t$ and $u$, as described in [1] (p. 282).

As in [1], we refer to $D_{T}$ and $B_{T}$ as the parabolic interior and parabolic boundary, respectively. Figure 2 gives a graphical 2-D representation of the interior and boundary. The inverse problem and its solution are stated here as a definition.

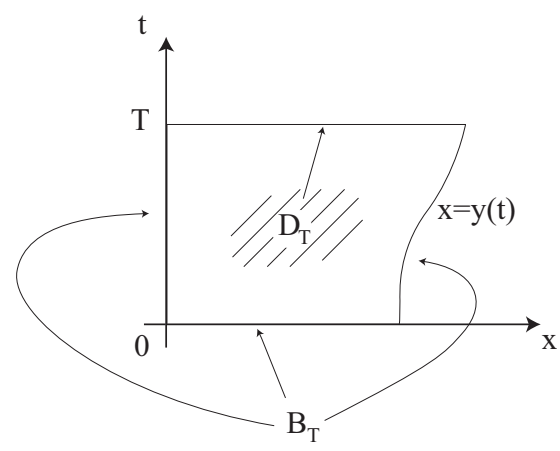

Figure 2. Picture of parabolic interior and boundary for free boundary problem.

Definition 1.1. A solution of (1) for a known function $y \in C^{\infty}[0, T]$, with all derivatives known, is a function $u=u(x, t)$ defined in $D_{T} \cup B_{T}$ such that $u_{x x}, u_{t} \in C\left(D_{T}\right), u$ is bounded, satisfies the conditions of (1) and $u \in C\left(D_{T} \cup B_{T}\right)$. 
The inverse problem is precisely a non-characteristic Cauchy problem with Cauchy data: $u(y(t), t)=0$, $u_{x}(y(t), t)=-\dot{y}(t)[7]$. Given $y(t)$ and a solution $u$ to the inverse problem, we have the initial profile $\psi(x)$ and the boundary control $h(t)$, both of which must be non-negative according to (1). Physically, the temperature everywhere in the liquid column should be non-negative; we will return to this point in the latter sections of the paper.

The flatness approach [3,4] for partial differential equations are a means of solving inverse problems. The explicit parametrization of the trajectories of the systems is the key to straightforward motion planning strategies that can incorporate optimization $[11,12]$.

Recent work on these parametrization include $[9,10]$ where approximate controllability of any initial condition to steady state is explored. The series expansion techniques used for heat equations have been used since the work of Gevrey, while inverse problems have been addressed as early as the work by Hill [7]. Specifically, Hill gives a complete solution to the inverse Stefan problem with a linear heat equation, i.e. $u_{t}=u_{x x}$. Recently a nonlinear heat equation over a constant spatial domain, with a quadratic reaction term, was examined by Rudolph and Lynch [10].

In this paper we combine the two issues: the free boundary (Stefan) problem with a quadratic nonlinearity. Using the work in [10] as a starting point, we prove convergence of a series solution. Then a detailed parametric study on the series radius of convergence is carried out. Moreover, we prove that the parametrization can indeed can be used for motion planning purposes; computation of the open loop motion planning is straightforward. Simulation results are given and we prove some important properties about the solution. Namely, a weak maximum principle is derived for the equation in (1), stating that the maximum is on the boundary. Also, we prove asymptotic positiveness of the solution, a physical requirement over the entire domain, as the transient time from one steady-state to another gets large.

The Stefan problem we consider is a first step towards a more complex problem for multidimensional reactiondiffusion systems investigated in [5], where three chemical species balance equations contain second order reaction terms. Extension of our approach to a fourth order radiation term, i.e. the Stefan-Boltzmann condition, is a subject for future work. A model with this condition arises in crystal growth furnaces, where a solid phase is surrounded by an infinite liquid phase. Note that in our work, either phase may be modelled with equivalent computations (after the appropriate sign changes). We feel the work presented here can highlight some of the difficulties and challenges of problems that arise in motion planning for crystal growth models.

\section{SERIES SOlutions AND CONVERGENCE}

\subsection{Outline}

In Section 2.2 we establish a lower bound on the radius of convergence of a series solution, denoted $\eta^{*}$, that depends upon the physical constants of the model $\rho$ and $\nu$. The radius $\eta^{*}$ also depends upon $M$ and $R$, the Gevrey constants of the function $\dot{y}(t)$. The definition of a Gevrey function is given implicitly in Theorem 2.1 (and also in [8]), from which it is clear that the associated constants ( $M$ and $R$ in this case) characterize the aggressiveness of the trajectories of the system. In Section 2.3 we make use of several lemmas to construct parametric expressions for lower bounds on the radius. Specifically, Lemma 2.4 allows us to bound $\eta^{*}$ from below by a unique root to a quartic polynomial that depends only upon $\rho, \nu, R$, where the root is compared to $M$ directly. In Lemma 2.5 we use a convexity argument to bound the root from Lemma 2.4 by an analytic expression that also depends only upon $\rho, \nu, R$. The two lemmas are then combined in Theorem 2.6 to give the main result regarding convergence of the series solution; namely, the radius of convergence is bounded by an analytic expression.

\subsection{Series solution}

Consider the series solution

$$
u(x, t)=\sum_{n=0}^{\infty} \frac{a_{n}(t)}{n !}[x-y(t)]^{n} .
$$


For the solution (2) to satisfy

$$
u_{t}=u_{x x}-\nu u_{x}-\rho u^{2}, \forall(x, t) \in D_{T}
$$

the $a_{n}(t)$ coefficients must satisfy the recurrence equation

$$
a_{n}=\dot{a}_{n-2}-a_{n-1} \dot{y}+\nu a_{n-1}+\rho \sum_{k=0}^{n-2}\left(\begin{array}{c}
n-2 \\
k
\end{array}\right) a_{n-2-k} a_{k}, \quad n \geq 2,
$$

with $a_{0}=0$ (arising from the $u(y(t), t)=0$ condition) and $a_{1}=-\dot{y}$ (arising from the Stefan condition $\left.-u_{x}(y(t), t)=\dot{y}(t)\right)$. From (3) it is clear that given $y(t)$, all the series coefficients $a_{n}(t)$ and therefore the temperature $u(x, t)$ and boundary conditions $h(t)$ and $\psi(x)$ are uniquely determined.

By majorizing the series in (2), we will prove that this solution converges absolutely. We now state the first of two main theorems in the paper. The proof makes use of two lemmas stated in the Appendix.

Theorem 2.1. Given that $\dot{y} \in C^{\infty}[0, T]$ is Gevrey of order $(\alpha-1)$ for $1 \leq \alpha \leq 2$, i.e.

$$
\exists M, R>0 \quad \text { such that }\left|y^{(l+1)}(t)\right| \leq M \frac{l !^{\alpha}}{R^{l}}, \forall l=0,1,2, \ldots, \forall t \in[0, T]
$$

the radius of convergence of the series has as a lower bound the unique positive root $\eta=\eta^{*}$ of the polynomial

$$
\left(\frac{\rho M}{2}\right) \eta^{3}+\left(\frac{1}{R}\right) \eta^{2}+\left(\frac{\nu+M}{2}\right) \eta-1=0
$$

Proof. By induction on $n$, we prove that for all $n=0,1,2, \ldots$, the coefficients satisfy the bound

$$
\left|a_{n}^{(l)}(t)\right| \leq \frac{M A^{n-1}}{R^{l+n-1}} \frac{(l+n) !^{\alpha}}{n !^{\alpha-1}}, \forall l=0,1,2, \ldots,
$$

for some $A>0$. The coefficient $a_{0}=0$ satisfies (5) trivially and we examine $n=1$ as the base case, since the recurrence is defined for $n \geq 2$. Namely, for $a_{1}=-\dot{y}$,

$$
\left|a_{1}^{(l)}(t)\right|=\left|y^{(l+1)}(t)\right| \leq M \frac{l !^{\alpha}}{R^{l}} \leq \frac{M}{R^{l}} l !^{\alpha}(l+1)^{\alpha},
$$

and the last inequality is strict when $l>0$. By inductive hypothesis, we assume now that (5) holds for all $i=0,1, \ldots, n-1$ and prove that it must also hold for $i=n$. Taking an absolute value and $l$ time derivatives of $(3)$, after the triangle inequality we have

$$
\left|a_{n}^{(l)}\right| \leq\left|a_{n-2}^{(l+1)}\right|+\nu\left|a_{n-1}^{(l)}\right|+\sum_{m=0}^{l}\left(\begin{array}{c}
l \\
m
\end{array}\right)\left|a_{n-1}^{(l-m)}\right|\left|y^{(m+1)}\right|+\rho \sum_{k=0}^{n-2} \sum_{r=0}^{l}\left(\begin{array}{c}
n-2 \\
k
\end{array}\right)\left(\begin{array}{c}
l \\
r
\end{array}\right)\left|a_{n-2-k}^{(r)}\right|\left|a_{k}^{(l-r)}\right| .
$$

The first two terms in (6) can be majorized using (5) as

$$
\begin{aligned}
& \left|a_{n-2}^{(l+1)}\right| \leq \frac{M A^{n-3}}{R^{l+n-2}} \frac{(l+n-1) !^{\alpha}}{(n-2) !^{\alpha-1}}=\frac{M A^{n-1}}{R^{l+n-1}} \frac{(l+n) !^{\alpha}}{n !^{\alpha-1}}\left[\frac{R}{A^{2}} \frac{(n(n-1))^{\alpha-1}}{(l+n)^{\alpha}}\right] \\
& \nu\left|a_{n-1}^{(l)}\right| \leq \nu \frac{M A^{n-2}}{R^{l+n-2}} \frac{(l+n-1) !^{\alpha}}{(n-1) !^{\alpha-1}}=\frac{M A^{n-1}}{R^{l+n-1}} \frac{(l+n) !^{\alpha}}{n !^{\alpha-1}}\left[\nu \frac{R}{A} \frac{n^{\alpha-1}}{(l+n)^{\alpha}}\right] .
\end{aligned}
$$


The third term in (6) is majorized as

$$
\begin{aligned}
\sum_{m=0}^{l}\left(\begin{array}{c}
l \\
m
\end{array}\right)\left|a_{n-1}^{(l-m)}\right|\left|y^{(m+1)}\right| & \leq \sum_{m=0}^{l}\left(\begin{array}{c}
l \\
m
\end{array}\right) \frac{M^{2} A^{n-2}}{R^{l+n-m-2} R^{m}} \frac{(l+n-m-1) !^{\alpha} m !^{\alpha}}{(n-1) !^{\alpha-1}} \\
& =\frac{M A^{n-1}}{R^{l+n-1}} \frac{(l+n) !^{\alpha}}{n !^{\alpha-1}}\left[\frac{M R}{A} \frac{n !^{\alpha-1}}{(l+n) !^{\alpha}} \frac{1}{(n-1) !^{\alpha-1}} \sum_{m=0}^{l}\left(\begin{array}{c}
l \\
m
\end{array}\right)(l+n-m-1) !^{\alpha} m !^{\alpha}\right] .
\end{aligned}
$$

Using Lemma A.1 and Lemma A.2, we can bound the term

$$
\sum_{m=0}^{l}\left(\begin{array}{c}
l \\
m
\end{array}\right)(l+n-m-1) !^{\alpha} m !^{\alpha} \leq\left[\sum_{m=0}^{l}\left(\begin{array}{c}
l \\
m
\end{array}\right)(l+n-m-1) ! m !\right]^{\alpha}=\left[\frac{(n-1) !(n+l) !}{n !}\right]^{\alpha},
$$

resulting in

$$
\sum_{m=0}^{l}\left(\begin{array}{c}
l \\
m
\end{array}\right)\left|a_{n-1}^{(l-m)}\right|\left|y^{(m+1)}\right| \leq \frac{M A^{n-1}}{R^{l+n-1}} \frac{(l+n) !^{\alpha}}{n !^{\alpha-1}}\left[\frac{M R}{A n}\right]
$$

The fourth (nonlinear) term in (6) is majorized as

$$
\begin{gathered}
\rho \sum_{k=0}^{n-2} \sum_{r=0}^{l}\left(\begin{array}{c}
n-2 \\
k
\end{array}\right)\left(\begin{array}{l}
l \\
r
\end{array}\right)\left|a_{n-2-k}^{(r)}\right|\left|a_{k}^{(l-r)}\right| \leq \rho \sum_{k=0}^{n-2} \sum_{r=0}^{l}\left(\begin{array}{c}
n-2 \\
k
\end{array}\right)\left(\begin{array}{l}
l \\
r
\end{array}\right) \frac{M^{2} A^{n-4}}{R^{l+n-4}} \frac{(n+r-k-2) !^{\alpha}(l+k-r) !^{\alpha}}{(n-k-2) !^{\alpha-1} k !^{\alpha-1}} \\
\quad \leq \frac{M A^{n-1}}{R^{l+n-1}} \frac{(l+n) !^{\alpha}}{n !^{\alpha-1}}\left[\frac{\rho M R^{3}}{A^{3}} \frac{n !^{\alpha-1}}{(l+n) !^{\alpha}} \sum_{k=0}^{n-2}\left(\begin{array}{c}
n-2 \\
k
\end{array}\right) \frac{1}{(n-k-2) !^{\alpha-1} k !^{\alpha-1}}\left\{\sum_{r=0}^{l}\left(\begin{array}{c}
l \\
r
\end{array}\right)(n+r-k-2) !(l+k-r) !\right\}^{\alpha}\right]
\end{gathered}
$$

where the last inequality makes use of Lemma A.2. Using Lemma A.1 we have

$$
\begin{aligned}
& \sum_{k=0}^{n-2}\left(\begin{array}{c}
n-2 \\
k
\end{array}\right) \frac{1}{(n-k-2) !^{\alpha-1} k !^{\alpha-1}}\left\{\sum_{r=0}^{l}\left(\begin{array}{l}
l \\
r
\end{array}\right)(n+r-k-2) !(l+k-r) !\right\}^{\alpha} \\
& \quad=\sum_{k=0}^{n-2}\left(\begin{array}{c}
n-2 \\
k
\end{array}\right) \frac{1}{(n-k-2) !^{\alpha-1} k !^{\alpha-1}}\left\{\frac{k !(n-k-2) !(n+l-1) !}{(n-1) !}\right\}^{\alpha} \\
& \quad=\sum_{k=0}^{n-2}\left(\begin{array}{c}
n-2 \\
k
\end{array}\right)(n-k-2) ! k !\left\{\frac{(n+l-1) !}{(n-1) !}\right\}^{\alpha}=\sum_{k=0}^{n-2}(n-2) !\left\{\frac{(n+l-1) !}{(n-1) !}\right\}^{\alpha} \\
& =(n-1)(n-2) !\left\{\frac{(n+l-1) !}{(n-1) !}\right\}^{\alpha}=\frac{(n+l-1) !^{\alpha}}{(n-1) !^{\alpha-1}},
\end{aligned}
$$

resulting in

$$
\begin{aligned}
\rho \sum_{k=0}^{n-2} \sum_{r=0}^{l}\left(\begin{array}{c}
n-2 \\
k
\end{array}\right)\left(\begin{array}{c}
l \\
r
\end{array}\right)\left|a_{n-2-k}^{(r)}\right|\left|a_{k}^{(l-r)}\right| & \leq \frac{M A^{n-1}}{R^{l+n-1}} \frac{(l+n) !^{\alpha}}{n !^{\alpha-1}}\left[\frac{\rho M R^{3}}{A^{3}} \frac{n !^{\alpha-1}}{(l+n) !^{\alpha}} \frac{(n+l-1) !^{\alpha}}{(n-1) !^{\alpha-1}}\right] \\
& =\frac{M A^{n-1}}{R^{l+n-1}} \frac{(l+n) !^{\alpha}}{n !^{\alpha-1}}\left[\frac{\rho M R^{3}}{A^{3} n}\left(\frac{n}{n+l}\right)^{\alpha}\right]
\end{aligned}
$$


Collecting the terms for (6) and noticing that for $n \geq 1, l \geq 0$, and $\alpha \geq 0,\left[\frac{n}{n+l}\right]^{\alpha} \leq 1$ holds, we have

$$
\left|a_{n}^{(l)}\right| \leq \frac{M A^{n-1}}{R^{l+n-1}} \frac{(l+n) !^{\alpha}}{n !^{\alpha-1}}\left[\frac{R}{A^{2}} \frac{(n-1)^{\alpha-1}}{n}+\frac{(\nu+M) R}{A n}+\frac{\rho M R^{3}}{A^{3} n}\right] .
$$

The terms in the square brackets are bounded as

$$
\max _{n \geq 2, \alpha \in[1,2]} \frac{(n-1)^{\alpha-1}}{n}=\left.\frac{(n-1)^{1}}{n}\right|_{(n \geq 2)} \leq 1, \quad \max _{n \geq 2} \frac{1}{n}=\frac{1}{2} .
$$

With these bounds, we have

$$
\left|a_{n}^{(l)}\right| \leq \frac{M A^{n-1}}{R^{l+n-1}} \frac{(l+n) !^{\alpha}}{n !^{\alpha-1}}\left[\frac{1}{R}\left(\frac{R}{A}\right)^{2}+\frac{(\nu+M)}{2}\left(\frac{R}{A}\right)+\frac{\rho M}{2}\left(\frac{R}{A}\right)^{3}\right] .
$$

Given $(M, R, \rho, \nu)$, the parameter $A$ is chosen such that

$$
\left[\frac{1}{R}\left(\frac{R}{A}\right)^{2}+\frac{(\nu+M)}{2}\left(\frac{R}{A}\right)+\frac{\rho M}{2}\left(\frac{R}{A}\right)^{3}\right]=1,
$$

implying that (5) is proven by induction. Using (5) and the Cauchy-Hadamard formula, the radius of convergence is given by

$$
\frac{1}{\lim \sup _{n \rightarrow \infty}\left|a_{n} / n !\right|^{1 / n}} \geq\left[\lim _{n \rightarrow \infty}\left|\frac{M A^{n-1}}{R^{n-1}}\right|^{1 / n}\right]^{-1}=\lim _{n \rightarrow \infty} \frac{R}{A}\left[\frac{A}{M R}\right]^{1 / n}=\frac{R}{A} .
$$

Denoting this lower bound on the radius of convergence as $\eta \equiv R / A$ and substituting into (7) yields (4). Existence and uniqueness of the positive root $\eta=\eta^{*}$ are now proven. First, given $(M, R, \nu, \rho)>0$ define the positive, analytic and strictly increasing function $\eta \mapsto f(\eta)$ as

$$
f(\eta)=\left(\frac{\rho M}{2}\right) \eta^{3}+\left(\frac{1}{R}\right) \eta^{2}+\left(\frac{\nu+M}{2}\right) \eta
$$

The positive root $\eta^{*}$ of the equation $f\left(\eta^{*}\right)-1=0$ exists and is unique since $(f(\cdot)-1)(\eta)$ is analytic and strictly increases from -1 to $+\infty$ as $\eta$ grows from 0 to $+\infty$.

Remark 2.2. We give here analytic expressions of the first five coefficients of the series (2) so one can see how the successive derivatives of $y$ appear

$$
\begin{aligned}
& a_{1}=-\dot{y} \\
& a_{2}=-\dot{y}(\nu+\dot{y}) \\
& a_{3}=-\ddot{y}+\dot{y}^{3}-\nu^{2} \dot{y} \\
& a_{4}=-\ddot{y}(2 \nu+\dot{y})-\dot{y}^{4}+\nu \dot{y}^{3}+\left(\nu^{2}+2 \rho\right) \dot{y}^{2}-\nu^{3} \dot{y} \\
& a_{5}=-y^{(3)}-3 \nu^{2} \ddot{y}+\dot{y}^{5}-2 \nu \dot{y}^{4}+4 \rho \dot{y}^{3}+\left(4 \ddot{y}+2 \nu\left(\nu^{2}+4 \rho\right)\right) \dot{y}^{2}+\left(\nu \ddot{y}^{2}-\nu^{4}\right) \dot{y}
\end{aligned}
$$




\subsection{Parameterizations of radius of convergence}

This section is concerned with constructing parametric lower bounds on $\eta^{*}$. We first derive by an easy calculation a lower bound that is suitable for most values of the physical parameters $\rho$ and $\nu$. This bound is then complemented with another lower bound that is more tedious to derive but is less conservative for specific values of the physical parameters, namely when $\rho$ is large and $\nu$ is small.

The first bound is achieved using the following lemma:

Lemma 2.3. For all $a, b, c$ strictly positive real parameters, the unique positive root $\eta^{0}$ of

$$
a \eta^{3}+b \eta^{2}+c \eta-1=0
$$

is lower bounded by

$$
\frac{-c+\sqrt{c^{2}+4(a / c+b)}}{2(a / c+b)} .
$$

Proof. The function $\eta \mapsto a \eta^{3}+b \eta^{2}+c \eta-1$ is analytic and strictly increases from -1 to $+\infty$ as $\eta$ grows from 0 to $+\infty$. Define $h_{1}(\eta)=a \eta^{3}+b \eta^{2}, h_{2}(\eta)=1-c \eta$. The graphs of $h_{1}$ and $h_{2}$ intersect at $\eta^{0}$. Since $h_{1}>0$ on $] 0,+\infty\left[\right.$ it is clear that $\eta^{0}<1 / c$.

On $] 0,1 / c\left[\right.$ it is easy to check that $h_{1}(\eta)<(a / c+b) \eta^{2}$. On this interval $h_{1}$ is a strictly increasing function while $h_{2}$ is strictly decreasing. Let $\hat{\eta}$ be the unique positive root of $(a / c+b) \eta^{2}=1-c \eta$ we get that $h_{1}(\hat{\eta})<h_{2}(\hat{\eta})$ which yields $\hat{\eta}<\eta^{0}$. Finally

$$
\eta^{0}>\frac{-c+\sqrt{c^{2}+4(a / c+b)}}{2(a / c+b)} .
$$

When $a=\rho M / 2, b=1 / R$ and $c=(\nu+M) / 2$, it is clear that $\eta^{0}$ corresponds to $\eta^{*}$.

The second lower bound is derived from the following lemmas:

Lemma 2.4. Given $R, \nu, \rho>0$, define the function $(\eta, M) \mapsto f(\eta, M)$ as

$$
f(\eta, M)=\left(\frac{\rho M}{2}\right) \eta^{3}+\left(\frac{1}{R}\right) \eta^{2}+\left(\frac{\nu+M}{2}\right) \eta .
$$

Let $M^{*}$ be the unique positive root of $f\left(M^{*}, M^{*}\right)=1$. Then, for any given $M$ with $0<M \leq M^{*}$, the root $\eta^{*}$ of $f\left(\eta^{*}, M\right)=1$ satisfies $\eta^{*} \geq M^{*}$.

Proof. The positive root $M^{*}$ of the equation $f\left(M^{*}, M^{*}\right)-1=0$ exists and is unique since $(f(\cdot)-1)(M, M)$ is analytic and strictly increases from -1 to $+\infty$ as $M$ grows from 0 to $+\infty$. Since $f$ is a strictly increasing function of $\eta$ and $M, f\left(\eta^{*}, M\right)=1=f\left(M^{*}, M^{*}\right)$ with $M \leq M^{*}$ implies that $M^{*} \leq \eta^{*}$.

From the lemma, $M=M^{*}$ is the unique positive root of the polynomial

$$
\left(\frac{\rho}{2}\right) M^{4}+\frac{1}{2}\left(\frac{R+2}{R}\right) M^{2}+\left(\frac{\nu}{2}\right) M-1=0
$$

giving a lower bound on the radius of convergence provided $M \leq M^{*}$. The next lemma gives a strict lower bound on $M^{*}$ using a convexity argument. Defining the functions

$$
g_{1}(M)=a M^{4}+b M^{2}, \quad g_{2}(M)=1-c M, \quad \text { where } a=\frac{\rho}{2}, b=\frac{1}{2}\left(\frac{R+2}{R}\right), c=\frac{\nu}{2},
$$

and it is clear that $g_{1}\left(M^{*}\right)=g_{2}\left(M^{*}\right)$. The functions, their intersection point $M^{*}$ and the line $c d M$ are shown in Figure 3, where $d=g_{1}(1 / c)>0$. 


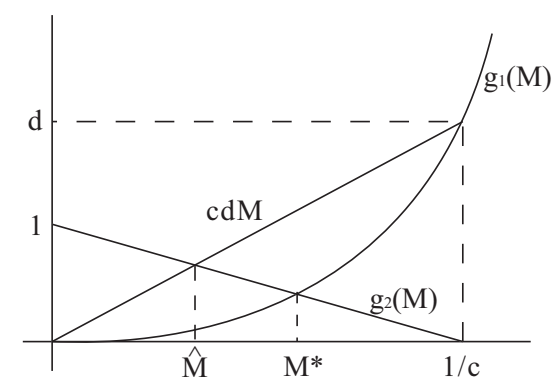

FIGURE 3. Illustration of convexity argument for strict lower bound on $M^{*}$.

The point $\hat{M}$ satisfies $c d \hat{M}=g_{2}(\hat{M})$, so after some algebra we have that

$$
d=\frac{a+c^{2} b}{c^{4}}, \quad \hat{M}=[c(d+1)]^{-1}=\left[c+b c^{-1}+a c^{-3}\right]^{-1} .
$$

Substituting in for the parameters and treating $\nu \mapsto \hat{M}(\nu)$ as a function given $(R, \rho)$, we have

$$
\hat{M}(\nu)=\left[\frac{\nu}{2}+\frac{4 \rho}{\nu^{3}}+\frac{R+2}{\nu R}\right]^{-1} .
$$

Lemma 2.5. Given $R, \rho>0$ and defining $\hat{\nu}=\arg \max _{\nu>0} \hat{M}(\nu)$, we have the following strict bounds on $M^{*}$ defined in Lemma 2.4:

$$
\begin{array}{ll}
M^{*}>\hat{M}(\nu), & \text { for all } \nu \in[\hat{\nu},+\infty[ \\
M^{*}>\hat{M}(\hat{\nu}), & \text { for all } \nu \in[0, \hat{\nu}[.
\end{array}
$$

Proof. We first compute $\hat{\nu}$ by taking the derivative of $\hat{M}$ with respect to $\nu$, yielding

$$
\frac{\mathrm{d} \hat{M}(\nu)}{\mathrm{d} \nu}=-\hat{M}(\nu)^{2}\left[\frac{1}{2}-\frac{12 \rho}{\nu^{4}}-\frac{R+2}{\nu^{2} R}\right] .
$$

Over the domain $\nu \in] 0,+\infty[, \hat{M}(\nu)>0$ so setting the derivative to zero to find the extremum requires the bracketed expression to be zero, which is equivalently

$$
\nu^{4}-\frac{2(R+2)}{R} \nu^{2}-24 \rho=0
$$

The unique positive solution to this squared-quadratic is

$$
\hat{\nu}=\left[\left(\frac{R+2}{R}\right)+\left[\left(\frac{R+2}{R}\right)^{2}+24 \rho\right]^{1 / 2}\right]^{1 / 2} .
$$

The second derivative at $\hat{\nu}$ is negative, verifying that the extremum is indeed a maximum. Observe that for all $R>0$ and $\rho \geq 0, \hat{\nu} \geq \sqrt{2}$. Also, $\hat{\nu}=\sqrt{2}$ if and only if $(R, \rho)=(+\infty, 0)$. For a given $(R, \rho)>0, g_{1}(M)$ is a strictly convex function, i.e.

$$
\left.c d M>g_{1}(M), \quad \forall M \in\right] 0,1 / c[.
$$


It is clear that $M^{*}$ and $\hat{M}$ are in the domain $] 0,1 / c\left[\right.$. By the convexity of $g_{1}$ then,

$$
c d M^{*}>g_{1}\left(M^{*}\right)=1-c M^{*} \Rightarrow M^{*}>[c(d+1)]^{-1}=\hat{M}(\nu)
$$

which holds for all $\nu \in] 0,+\infty\left[\right.$. The trouble with $\hat{M}(\nu)$ as a bound for $M^{*}$ over $\nu \in[0, \hat{\nu}[$ is that as $\nu \rightarrow 0$, $\hat{M}(\nu) \rightarrow 0$ while $M^{*}$ is increasing up to the solution of the equation $g_{2}(M)=1$. Since we know $M^{*}>\hat{M}(\nu)$ for $\nu=\hat{\nu}$ and $M^{*}$ is increasing as $\nu$ decreases from $\hat{\nu}$ to 0 , we can (conservatively) choose the lower bound on $M^{*}$ to remain at the maximum $\hat{M}(\hat{\nu})$ for all $\nu \in[0, \hat{\nu}[$.

We now give the main analytic result of the paper regarding convergence of the proposed solution, making use of the two lower bounds derived above.

Theorem 2.6. Given $\nu, \rho>0$ and assuming that $\dot{y} \in C^{\infty}[0, T]$ is Gevrey of order $(\alpha-1)$ for $1 \leq \alpha \leq 2$, i.e.

$$
\exists M, R>0 \quad \text { such that }\left|y^{(l+1)}(t)\right| \leq M \frac{l^{!^{\alpha}}}{R^{l}}, \forall l=0,1,2, \ldots,
$$

the radius of convergence of the series (2) is greater than

$$
\hat{\eta}=\frac{-R(\nu+M)^{2}+\sqrt{R^{2}(\nu+M)^{4}+16\left(\rho M R^{2}(\nu+M)+R(\nu+M)^{2}\right)}}{4(\rho R M+\nu+M)} .
$$

Furthermore, if $M^{*} \geq M, M^{*}$ given by (10), then the radius of convergence of the series (2) is greater than the following quantities

$$
\left.\begin{array}{l}
\hat{M}(\nu), \quad \text { for all } \nu \in[\hat{\nu},+\infty[ \\
\hat{M}(\hat{\nu}), \quad \text { for all } \nu \in[0, \hat{\nu}[
\end{array}\right\}
$$

where $\hat{M}$, $\hat{\nu}$ are given by (11), (12), respectively.

Proof. We get the first lower bound (13) from Lemma 2.3 where we make $a=\rho M / 2, b=1 / R, c=(\nu+M) / 2$.

From Theorem 2.1, $\eta^{*}$ is a lower bound on the radius of convergence. Since $M^{*} \geq M$ by assumption we can apply Lemma 2.4, and with Lemma 2.5 we have

$$
\eta^{*} \geq M^{*}>\hat{M}
$$

giving the stated result in (14).

Remark 2.7. Let us here characterize the different lower bounds on the radius of convergence, given the parameter values $\rho, \nu, M$ and $R$. Calculating $\eta^{*}$ numerically will of course result in the least conservative bound. Of the two analytic lower bounds, equation (13) is less conservative than (14) for most values of the physical parameters $\rho$ and $\nu$. Only when $\rho$ is large and $\nu<\hat{\nu}$ does (14) become less conservative. Specifically, in that case, $\hat{\eta} \propto \rho^{-1 / 2}$ and $\hat{M} \propto \rho^{-1 / 4}$ and thus $\hat{\eta}$ approaches zero faster than $\hat{M}$ as $\rho$ approaches infinity. Numerical comparisons between these bounds are given in the simulation studies in Section 3.2.

\section{Properties of SOlution APplicAtion}

The output domain is $y:[0, T] \rightarrow \mathbb{R}$ and we define $y(t)=\phi(t / T)$. Then, given $\phi:[0,1] \rightarrow \mathbb{R}$ with Gevrey bounds

$$
\exists M_{\phi}, R_{\phi}>0 \quad \text { such that }\left|\phi^{(l+1)}(t)\right| \leq M_{\phi} \frac{l !^{\alpha}}{R_{\phi}^{l}}, \forall l=0,1,2, \ldots
$$


and observing that

$$
y^{(l)}(t)=\frac{1}{T^{l}} \phi^{(l)}(t / T)
$$

the Gevrey bounds on $y(t)$ become,

$$
\left|y^{(l+1)}(t)\right| \leq \frac{M_{\phi}}{T} \frac{l !^{\alpha}}{\left(R_{\phi} T\right)^{l}}=M \frac{l !^{\alpha}}{R^{l}}, \quad M \equiv M_{\phi} / T, R \equiv R_{\phi} T .
$$

Thus, given the function $\phi:[0,1] \rightarrow \mathbb{R}$, the conditions of Theorem 2.6 can be checked by substituting $M=M_{\phi} / T$ and $R=R_{\phi} T$.

In the following, we give numerical simulations and discuss theoretical properties about the results. The time bound parameter $T$ is shown to be important for guaranteeing desirable properties of the solution. Intuition about the physical problem suggests that smaller $T$ will require more aggressive trajectories for steady-state to steady-state boundary control. Using the recurrence relation, we prove that as $T$ grows we can approximate the series solution to second order by an analytic expression. The expression guarantees that the temperature profile in the liquid column remains above the thawing point of ice ( 0 degrees which is the temperature of the interface).

\subsection{Physical aspects of solution}

The classical Stefan problem assumes the given initial profile satisfies $\psi(x) \geq 0$ in $B_{T}^{2}$ and the boundary control satisfies $h(t) \geq 0$ in $B_{T}^{1}$. In this case, from a Weak Maximum/Minimum Principle, the temperature in $D_{T}$ never exceeds or drops below the temperature of the column on $B_{T}$ (see Sect. 1.6 in [1]). Thus, it is a given that the temperature everywhere is non-negative since $u \geq 0$ in $B_{T}$.

For the inverse nonlinear problem, we are faced with guaranteeing that our solution satisfies this physical requirement that the temperature in the liquid column never drops below the freezing point. The specific Gevrey function chosen for $\dot{y}(t)$ will be required to satisfy certain properties. In Section 17.2 of [1], $y(t)$ is shown to be an increasing function and strictly increasing if $\psi$ or $h$ are nonzero in every neighborhood of $t=0$. Of course, we cannot expect a converse-like result without a similar assumption imposed on $y(t)$. Specifically we assume that given any $\epsilon \in] 0,1 / 2[$,

$$
\dot{y}(t)>0, \forall t \in[T \epsilon, T(1-\epsilon)],
$$

and $\dot{y}(t)=0 \Longleftrightarrow t=0$ or $t=T$. Moreover, for steady-state behavior we assume that

$$
y(0)=L, y(T)=L+\Delta L, \quad \text { where } L, \Delta L \in \mathbb{R}^{+}, \quad \text { and } y^{(m)}(0)=y^{(m)}(T)=0, \forall m=1,2,3, \ldots
$$

From (3), $a_{n}(0)=a_{n}(T)=0$, for all $n=0,1,2, \ldots$ As a result, $u(x, 0)=u(x, T)=0$. So $u=0$ on the open line $\partial D_{T} \equiv\{(x, T): 0<x<y(T)\}$ and on $B_{T}^{2}$ and $B_{T}^{3}$.

Proving that the temperature satisfies $u(x, t) \geq 0$ for all $(x, t)$ in $D_{T}-\partial D_{T}$ and for all $t$ in $B_{T}^{1}$ would thus assure that $u(x, t) \geq 0$ in $D_{T} \cup B_{T}$. Given a Weak Minimum Principle, we could assure the same if we proved $u(x, t) \geq 0$ in $B_{T}^{1}$. We do not establish a Weak Minimum Principle here. Instead we derive a Weak Maximum Principle, that serves as a sanity check for numerical experiments (i.e. the interior temperature should never exceed the maximum boundary temperature) and focus on a detailed analytic study. It is shown that approximate steady-state to steady-state boundary control can guarantee non-negativeness of the temperature in the entire domain.

\subsection{Numerical simulations}

For practical purposes of course the series solution (2) is truncated for implementation. Specifically, for $y(t)$ defined by the function given in Appendix B, we here take the first 10 terms to approximate the solution $u(x, t)$. 
Steady-state to steady-state simulations are considered and so, as shown above, $\psi \equiv 0$. In Figure 4 we show the temperature profile for $T=100, \rho=1.5, \nu=0.5, L=1$, and $L+\Delta L=2$. For this case, we compute $\eta^{*}=2.1849$, which guarantees convergence of the desired domain. To give a case where the more conservative, analytic lower bounds (13) and (14) in Theorem 2.6 guarantee convergence, see Figure 5. For this temperature profile, the parameters are $T=100, \rho=1.2, \nu=0.5, L=0.25$, and $L+\Delta L=0.5$. The analytic expressions yield $\hat{\eta}=2.5165$ and $\hat{M}=0.50302$. For this case, we also computed $\eta^{*}=2.586$ showing the conservatism of (14) quantitatively.

The lower bounds on the radius of convergence can also be compared analytically as $T$ becomes large. In that case, $\eta^{*}$ and $\hat{\eta}$ approach $2 / \nu$, while $\hat{M}$ approaches $\left(\nu / 2+4 \rho / \nu^{3}+1 / \nu\right)^{-1}$, again displaying the relative conservatism of (14). In both simulations, the time bound $T$ was chosen large enough such that the temperature in the column remained non-negative. An asymptotic analysis of how large $T$ needs to be to ensure nonnegativity is detailed in Section 3.4.

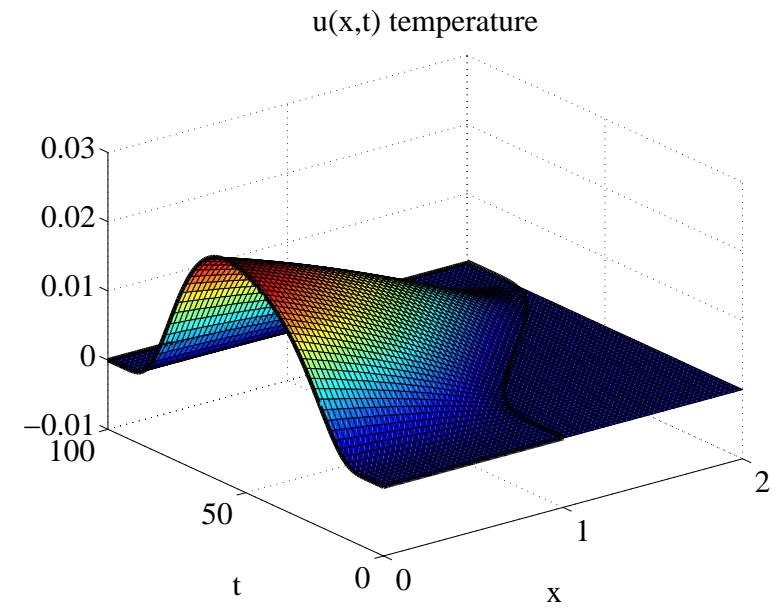

Figure 4. Temperature profile for transition from column length 1 to 2.

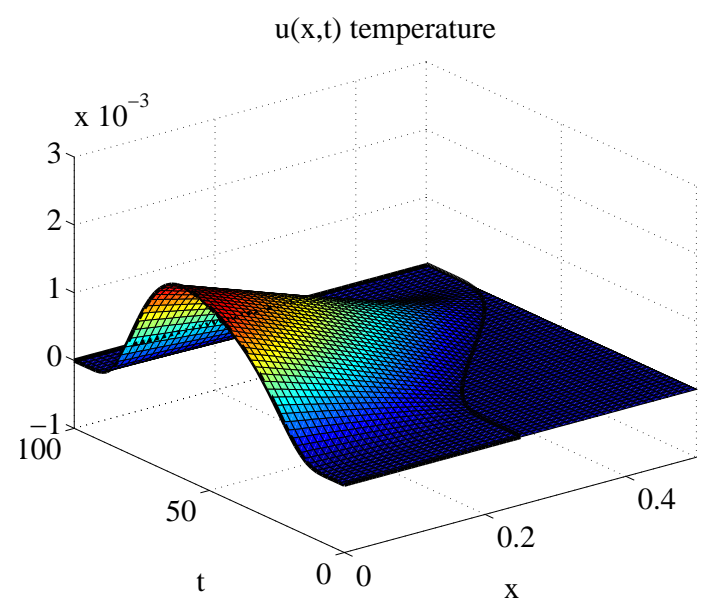

Figure 5. Temperature profile for transition from column length 0.25 to 0.5 . 


\subsection{The Weak Maximum Principle (WMP)}

A powerful tool for the study of linear initial-boundary-value problems is the Weak Maximum Principle [1]. We now state this tool as it applies to (1).

Theorem 3.1. For a solution $u$ of $u_{t}=u_{x x}-\nu u_{x}-\rho u^{2}$ in a bounded $D_{T}$, with $\nu, \rho \geq 0$, which is continuous in $D_{T} \cup B_{T}$,

$$
\max _{D_{T} \cup B_{T}} u=\max _{B_{T}} u
$$

Proof. The proof follows along the lines of the proof of the Weak Maximum Principle (Th. 1.6.1) for $u_{t}=u_{x x}$ given in [1]. From Definition 1.1, the value of $u$ is bounded in $D_{T} \cup B_{T}$ and we denote this bound $|u| \leq U$. Define

$$
v(x, t)=u(x, t)+\varepsilon \exp [W(x-C)]
$$

over $D_{T} \cup B_{T}$, where $\varepsilon, W>0$ and $|y(t)| \leq C \equiv L+\Delta L$. Then $v$ assumes its maximum on $B_{T}$. Otherwise, there exists a point $\left(x_{0}, t_{0}\right) \in D_{T}$ such that

$$
\max _{D_{T} \cup B_{T}} v=v\left(x_{0}, t_{0}\right)
$$

Hence at $\left(x_{0}, t_{0}\right) \in D_{T}$,

$$
\mathcal{L}(v) \equiv v_{x x}-v_{t}-\nu v_{x}-\rho v^{2} \leq 0
$$

since $0<x<y(t), 0<t \leq T$ in $D_{T}$ and

$$
\begin{aligned}
& v_{x x}\left(x_{0}, t_{0}\right) \leq 0 \text { (at a maximum), } \\
& v_{x}\left(x_{0}, t_{0}\right)=0 \text { (at an extremum), } \\
& v_{t}\left(x_{0}, t_{0}\right) \geq 0 \text { (at a maximum over a right-closed domain), }
\end{aligned}
$$

where $v_{t}\left(x_{0}, t_{0}\right)$ may be positive in $D_{T}$ only when $t_{0}=T$. However, throughout $D_{T}$,

$$
\begin{aligned}
\mathcal{L}(v)= & u_{x x}-u_{t}-\nu u_{x}-\rho\{u+\varepsilon \exp [W(x-C)]\}^{2} \\
& +\varepsilon W^{2} \exp [W(x-C)]-\nu \varepsilon W \exp [W(x-C)] \\
= & \varepsilon W(W-\nu) \exp [W(x-C)]-\rho\left\{2 u \varepsilon \exp [W(x-C)]+\varepsilon^{2} \exp [2 W(x-C)]\right\} \\
= & \varepsilon \exp [W(x-C)]\{W(W-\nu)-\rho(2 u+\varepsilon \exp [W(x-C)])\} \\
& >\varepsilon \exp [-W C]\{W(W-\nu)-\rho(2 U+\varepsilon)\},
\end{aligned}
$$

where the inequality follows from $0<x<C$ in $D_{T}$, which implies $\left.\exp (W(x-C)) \in\right] \exp (-W C), 1[$, and from choosing $W$ large enough, namely such that

$$
W(W-\nu)>\rho(2 U+\varepsilon)
$$

As a result, $\mathcal{L}(v)>0$. Thus by contradiction $v$ assumes its maximum value on $B_{T}$.

Since $u \leq v$ on $D_{T} \cup B_{T}$ and $v \leq \max _{B_{T}} v$,

$$
u \leq \max _{B_{T}} v \leq \max _{B_{T}} u+\varepsilon \max _{B_{T}} \exp [W(x-C)] .
$$


As $\varepsilon$ can be chosen arbitrarily small,

$$
u \leq \max _{B_{T}} u .
$$

The Weak Maximum Principle is typically accompanied by its dual, the Weak Minimum Principle, as the latter principle can be directly derived from the former by replacing $u$ with $(-u)$ in the heat equation. With a quadratic nonlinearity of course, $(-u)$ does not satisfy the same equation so we do not have a dual principle as directly. The simulation studies have indicated that there is a Weak Minimum Principle, but we do not pursue the theoretical version here. In the next section, to assure positivity of the temperature in the liquid column, we examine asymptotic behavior of the solution $u$ as our time bound $T$ is made large.

\subsection{Asymptotics of the solution}

In this section, it is shown that positivity of the temperature everywhere in the column can be guaranteed with as much resolution, up to the boundaries of the domain, as desired given a sufficiently large $T$.

Lemma 3.2. Consider the problem (1) and series solution (2), where the output $y:[0, T] \rightarrow \mathbb{R}$ is defined as $y(t)=\phi(t / T)$, given $\phi:[0,1] \rightarrow \mathbb{R}$ satisfying $(15)$. When $T$ is large enough, the series is uniformly approximated to second order in $(1 / T)$ by

$$
-\frac{\dot{y}(t)}{\nu}(\exp [\nu(x-y(t))]-1) .
$$

Proof. First, we prove by induction that the recurrence equation (3) can be expressed as

$$
a_{n}=-\nu^{n-1} \dot{y}+\hat{F}_{n}\left(y^{(g(n))}, y^{(g(n)-1)}, \ldots, \dot{y}\right), \quad n \geq 1,
$$

where $\hat{F}_{n}$ is a multivariate polynomial, in $\dot{y}$ and its time derivatives up to order $g(n)$, that has no constant terms and no terms affine in $\dot{y}$. It is also clear from the (3) that $\hat{F}$ is time-invariant, i.e. $t$ does not appear explicitly. By induction it can be shown that $g(n) \in \mathbb{N}$ is given by $g(n)=1+\operatorname{floor}((n-1) / 2)$, which means that $g(n \pm 2)=g(n) \pm 1$. The base case $a_{1}=-\dot{y}$, and the next term $a_{2}=-\nu \dot{y}-\dot{y}^{2}$, satisfy (17). By inductive hypothesis we assume (17) holds for $i=2, \ldots, n-1$ and show that it also holds for $i=n$. From (3) we have

$$
\begin{aligned}
a_{n}= & \frac{\mathrm{d}}{\mathrm{d} t}\left[-\nu^{n-3} \dot{y}+\hat{F}_{n-2}\left(y^{(g(n-2))}, \ldots, \dot{y}\right)\right]+(\nu-\dot{y})\left[-\nu^{n-2} \dot{y}+\hat{F}_{n-1}\left(y^{(g(n-1))}, \ldots, \dot{y}\right)\right] \\
& +\rho \sum_{k=0}^{n-2}\left(\begin{array}{c}
n-2 \\
k
\end{array}\right)\left[-\nu^{n-3-k} \dot{y}+\hat{F}_{n-2-k}\left(y^{(g(n-2-k))}, \ldots, \dot{y}\right)\right]\left[-\nu^{k-1} \dot{y}+\hat{F}_{k}\left(y^{(g(k))}, \ldots, \dot{y}\right)\right] .
\end{aligned}
$$

Given the properties on $\hat{F}_{m}$, for any $m=1, \ldots, n-1$, we can rewrite it as

$$
\hat{F}_{m}\left(y^{(g(m))}, \ldots, \dot{y}\right)=\dot{y} \hat{F}_{m}^{1}\left(y^{(g(m))}, \ldots, \dot{y}\right)+\hat{F}_{m}^{2}\left(y^{(g(m))}, \ldots, \ddot{y}\right)
$$

where $\hat{F}_{m}^{1}$ and $\hat{F}_{m}^{2}$ have no constant terms. Observe that

$$
\frac{\mathrm{d}}{\mathrm{d} t} \hat{F}_{m}\left(y^{(g(m))}, \ldots, \dot{y}\right)=\ddot{y} \hat{F}_{m}^{1}\left(y^{(g(m))}, \ldots, \dot{y}\right)+\dot{y} \bar{F}_{m+2}^{1}\left(y^{(g(m+2))}, \ldots, \dot{y}\right)+\bar{F}_{m+2}^{2}\left(y^{(g(m+2))}, \ldots, \ddot{y}\right),
$$

where $\bar{F}_{m+2}^{i} \equiv \mathrm{d} / \mathrm{d} t\left(\hat{F}_{m}^{i}\right), i=1,2$. As $\hat{F}_{m}^{i}$ has no explicit dependence on $t, \bar{F}_{m+2}^{i}$ can have no constant terms. Thus, $\mathrm{d} / \mathrm{d} t\left(\hat{F}_{m}\left(y^{(g(m))}, \ldots, \dot{y}\right)\right)$ has no constant terms or terms that are affine in $\dot{y}$, and we rewrite it as

$$
\frac{\mathrm{d}}{\mathrm{d} t} \hat{F}_{m}\left(y^{(g(m))}, \ldots, \dot{y}\right)=\bar{F}_{m+2}\left(y^{(g(m+2))}, \ldots, \dot{y}\right) .
$$


Returning to the expression for $a_{n}$ and applying the previous result for $m=n-2$ we have

$$
\begin{aligned}
a_{n}= & {\left[-\nu^{n-3} \ddot{y}+\bar{F}_{n}\left(y^{(g(n))}, \ldots, \dot{y}\right)\right]-\nu^{n-1} \dot{y}+\nu^{n-2} \dot{y}^{2}+(\nu-\dot{y}) \hat{F}_{n-1}\left(y^{(g(n-1))}, \ldots, \dot{y}\right) } \\
& +\rho \sum_{k=0}^{n-2}\left(\begin{array}{c}
n-2 \\
k
\end{array}\right)\left[-\nu^{n-3-k} \dot{y}+\hat{F}_{n-2-k}\left(y^{(g(n-2-k))}, \ldots, \dot{y}\right)\right]\left[-\nu^{k-1} \dot{y}+\hat{F}_{k}\left(y^{(g(k))}, \ldots, \dot{y}\right)\right] \\
= & -\nu^{n-1} \dot{y}+\hat{F}_{n}\left(y^{(g(n))}, \ldots, \dot{y}\right),
\end{aligned}
$$

where we have defined $\hat{F}_{n}$ as the collected terms and it is clear that $\hat{F}$ also has no terms affine in $\dot{y}$ or constant terms. Note that we do not claim that collecting terms in the last step does not result in the cancellation of terms. We only prove that it is not possible to generate new terms that are affine in $\dot{y}$ or constant. This concludes that induction and proves (17).

The next step is rewrite (17) with $\hat{F}_{n}$ as a function of the given output $\phi$ and its derivatives, as

$$
a_{n}(t)=-\nu^{n-1} \dot{y}(t)+\hat{F}_{n}\left(\frac{\phi^{(g(n))}(t / T)}{T^{g(n)}}, \ldots, \frac{\dot{\phi}(t / T)}{T}\right), \quad n \geq 1, t \in[0, T] .
$$

Given the properties on the multivariate polynomial $\hat{F}$, the lowest order terms in $1 / T$ that are possible are $\dot{y}^{2}$ and $\ddot{y}$, which means that as $T \rightarrow+\infty, \hat{F}_{n}=O\left(1 / T^{2}\right)$ uniformly and

$$
a_{n}(t)=-\nu^{n-1} \dot{y}(t)+O\left(1 / T^{2}\right), \quad n \geq 1, t \in[0, T] .
$$

Using this as the recurrence equation for the series solution, we can write (2) as

$$
\begin{aligned}
u(x, t) & =-\frac{\dot{y}(t)}{\nu} \sum_{n=1}^{\infty} \frac{1}{n !}[\nu(x-y(t))]^{n}+O\left(1 / T^{2}\right) \sum_{n=1}^{\infty} \frac{1}{n !}[x-y(t)]^{n} \\
& =-\frac{\dot{y}(t)}{\nu}(\exp [\nu(x-y(t))]-1)+O\left(1 / T^{2}\right)(\exp [x-y(t)]-1) \\
& =-\frac{\dot{y}(t)}{\nu}(\exp [\nu(x-y(t))]-1)+O\left(1 / T^{2}\right),
\end{aligned}
$$

which concludes the proof.

We can visualize the implications of Lemma 3.2 by looking at the $a_{n}$ coefficients. Figure 6 shows the first ten coefficients for the parameter case study corresponding to Figure 4 with $T=10$, in which case the temperature in the liquid does go negative. Figure 7 shows the coefficients for $T$ increased to 100, corresponding exactly to the case in Figure 4. From the proof of the lemma, equation (17) implies that as $T$ increases the coefficients approach $a_{n} \simeq-\nu^{n-1} \dot{y}(t)=-\nu^{n-1} \dot{\phi}(t / T) / T$. So as $T$ increases, all of the coefficients approach the shape of $-\dot{y}$ (a negative definite, symmetric function) and decrease in amplitude, both trends observable from the figures.

The following result guarantees that up to arbitrarily small precision, we can achieve steady-state to statestate boundary control while maintaining a positive temperature in the entire liquid column, provided the upper bound on time $T$ is large enough.

Lemma 3.3. Consider the problem (1) and series solution (2), where we take $\nu>0$. The output $y:[0, T] \rightarrow \mathbb{R}$ is defined as $y(t)=\phi(t / T)$, given $\phi:[0,1] \rightarrow \mathbb{R}$ satisfying (15). Assume $\phi$ is a strictly increasing function, so $y(t)$ satisfies (16). Given any $1 / 2>\varepsilon_{t}>0$ and $L>\varepsilon_{x}>0$, there exists $T_{\min }>0$ such that for all $T>T_{\min }$ the temperature satisfies

$$
u(x, t)>0, \quad \forall(x, t) \in D_{T}^{\varepsilon} \cup B_{T}^{\varepsilon},
$$




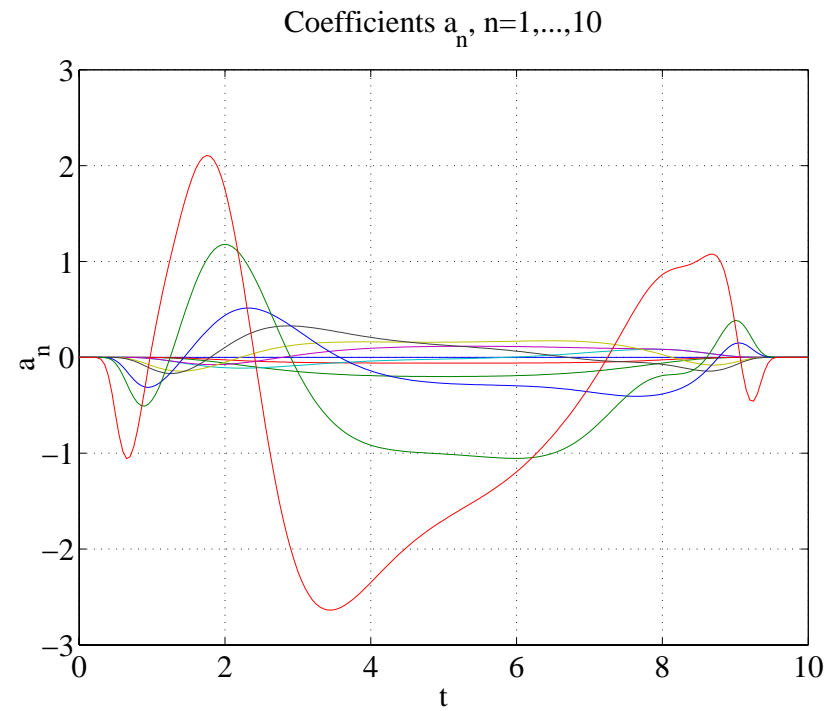

Figure 6. First ten $a_{n}$ coefficients for $T=10$.

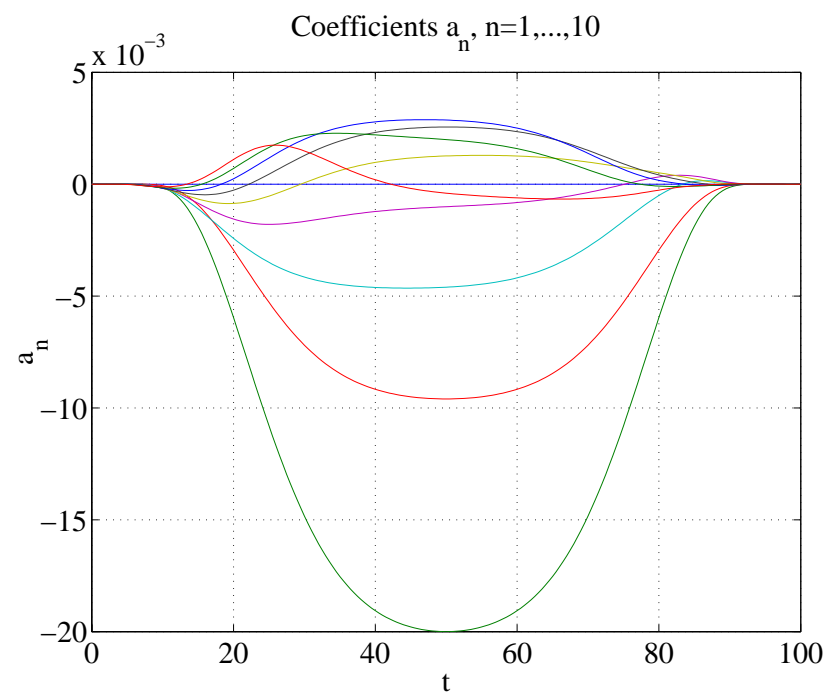

FiguRE 7. First ten $a_{n}$ coefficients for $T=100$.

where

$$
\begin{aligned}
D_{T}^{\varepsilon} \equiv & \left\{(x, t): 0<x<y(t)-\varepsilon_{x}, T \varepsilon_{t}<t \leq T\left(1-\varepsilon_{t}\right)\right\} \\
B_{T}^{\varepsilon} \equiv & \left\{(0, t): T \varepsilon_{t}<t \leq T\left(1-\varepsilon_{t}\right)\right\} \\
& \cup\left\{\left(x, T \varepsilon_{t}\right): 0 \leq x \leq y\left(T \varepsilon_{t}\right)-\varepsilon_{x}\right\} \\
& \cup\left\{\left(y(t)-\varepsilon_{x}, t\right): T \varepsilon_{t}<t \leq T\left(1-\varepsilon_{t}\right)\right\}
\end{aligned}
$$

A graphical 2-D representation of the $\varepsilon$ interior and boundary $D_{T}^{\varepsilon}, B_{T}^{\varepsilon}$ is given in Figure 8 . 


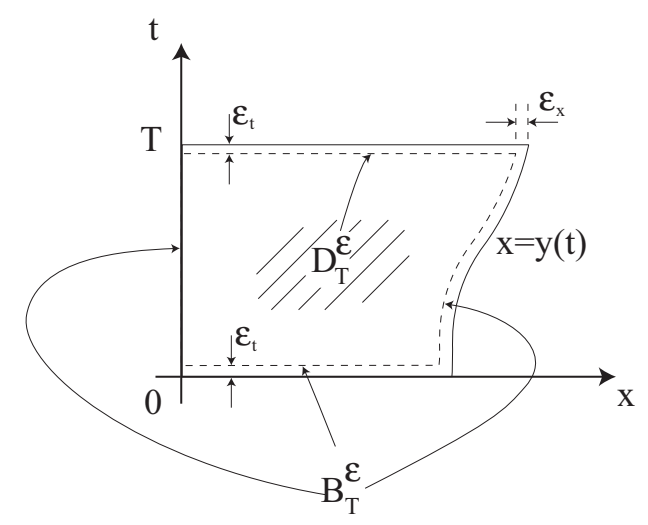

FiguRe 8. Picture of parabolic $\varepsilon$ interior and boundary for Stefan problem.

Proof. By assumption,

$$
\dot{\phi}(s)>0, \forall s \in\left[\varepsilon_{t},\left(1-\varepsilon_{t}\right)\right] .
$$

Define $\gamma \equiv \min _{s} \dot{\phi}(s)>0$, with $s \in\left[\varepsilon_{t},\left(1-\varepsilon_{t}\right)\right]$. Also, observe that

$$
x-\phi(t / T) \leq-\varepsilon_{x}<0, \quad \forall(x, t) \in D_{T}^{\varepsilon} \cup B_{T}^{\varepsilon} .
$$

Now, we apply Lemma 3.2 to get

$$
\begin{aligned}
u(x, t) & =-\frac{\dot{\phi}(t / T)}{T \nu}(\exp [\nu(x-\phi(t / T))]-1)+O\left(1 / T^{2}\right) \\
& \geq-\frac{\gamma}{T \nu}\left(\exp \left[-\nu \varepsilon_{x}\right]-1\right)+O\left(1 / T^{2}\right), \quad \forall(x, t) \in D_{T}^{\varepsilon} \cup B_{T}^{\varepsilon} .
\end{aligned}
$$

Since $\gamma, \nu, \varepsilon_{x}>0$, the term $-\gamma\left(\exp \left[-\nu \varepsilon_{x}\right]-1\right) / \nu$ is strictly positive. Therefore, there exists a $T_{\min }$ that depends on $\gamma\left(\right.$ i.e. on $\left.\varepsilon_{t}\right)$ and $\varepsilon_{x}$ such that for $T>T_{\min }$, the positive term dominates and $u(x, t)>0$ for all $(x, t) \in D_{T}^{\varepsilon} \cup B_{T}^{\varepsilon}$.

For the simulation parameters used for the case of Figure 4, we numerically investigated values for $\varepsilon_{t}$ as $T$ increases, with the results given in Table 1 . The values for $\varepsilon_{t}$ reported are lower bounds, meaning we (conservatively) estimate the largest distance from the boundary at which negative temperatures occur. The distance is a lower bound for the following reason: if for a given $\varepsilon_{t}$ we have a $T$ that satisfies Lemma 3.3, for any $\varepsilon>\varepsilon_{t}$, the same $T$ will result in a positive $u$ in $D_{T}^{\varepsilon} \cup B_{T}^{\varepsilon}$. The trend of the table is that as $\varepsilon_{t}$ decreases, $T$ must increase, as implied by Lemma 3.3. From the table, the simulation corresponding to Figure $4(T=100)$ is guaranteed to have positiveness up to $\varepsilon_{t}=0.058$.

TABLE 1. Estimation of $\varepsilon_{t}$ parameter for Lemma 3.3.

\begin{tabular}{|c|c|c|c|c|c|}
\hline$T$ & 5 & 10 & 50 & 100 & 500 \\
\hline$\varepsilon_{t}$ & 0.267 & 0.190 & 0.0851 & 0.0580 & 0.0217 \\
\hline
\end{tabular}

\section{Conclusions and Future WORK}

The problem treated in this paper includes two technical difficulties: the moving boundary and a quadratic reaction term. When combined, these issues make convergence substantially more difficult to study. We derived 
conservative results that can indeed be used in practice, as shown in the simulation section. We underlined that the solution we propose has to be used very carefully or the formal solution might not satisfy the physical requirement of the model (non-negativity of the temperature in the liquid phase).

An issue for future work is the study of approximate controllability of the model studied in this paper. Replacing the zero initial condition $u(x, 0)=0$ by an arbitrary initial condition $u(x, 0)=\psi(x)$, is it still possible to steer the system approximately to zero in finite time, i.e. $u(x, T) \approx 0$ ?

Usually, such a result arises from a projection of the initial condition onto the formal series expression (refer to [9]). Straightforward conditions are thus available for formal series of the form $u(x, t)=\sum_{i=0}^{\infty} y^{(n)}(t) \frac{x^{2 n}}{(2 n) !}$ using the fact that the set of polynomials $x^{2 n}, n \in \mathbb{N}$ is dense in the set of $L^{2}$ functions. In a simple manner, the conditions result in specified values for all the derivatives of $y$ at the initial time 0 .

In our case the series expansion is much different. Due to the moving boundary and the nonlinear effect, no simple identification between the $\left(a_{i}\right)$ coefficients and the derivatives of $y$ can be achieved. Moreover, both even and odd polynomials appear for most derivatives of $y$, ruling out classical density results, e.g. Stone-Weierstrass theorem [15]. All this makes the situation more convoluted and difficult to handle. Gathering terms it seems possible to derive solvable conditions in terms of the successive derivatives of $y$ and some projections of the initial condition $\psi(x)$. This point is currently under investigation but we sketch an explicit procedure here.

\section{Treating a non zero initial condition}

Let us sketch here how one can derive the conditions on the successive derivatives of $y$. From the series solution (2) and remembering that $a_{0}=0$ from the boundary condition, one gets

$$
\begin{aligned}
\psi(x)=u(x, 0) & =\sum_{n=1}^{\infty} \frac{a_{n}(0)}{n !}(x-y(0))^{n} \\
& =\sum_{n=1}^{\infty} a_{2 n-1}(0) \frac{(x-y(0))^{2 n-1}}{(2 n-1) !}\left(1+\frac{a_{2 n}(0)}{2 n a_{2 n-1}(0)}(x-y(0))\right)
\end{aligned}
$$

where the last line assumes that every ratio $\frac{a_{2 n}}{a_{2 n-1}}$, for $n=1,2, \ldots$, has a limit around zero.

Denoting $P_{n}(x)=\frac{(x-y(0))^{2 n-1}}{(2 n-1) !}\left(1+\frac{a_{2 n}(0)}{2 n a_{2 n-1}(0)}(x-y(0))\right)$, the initial condition reads

$$
\psi(x)=\sum_{n=1}^{\infty} a_{2 n-1}(0) P_{n}(x)
$$

This idea is then to project $\psi$ onto a basis of such polynomials. To do so it is necessary to derive an orthonormal set of polynomials from the set $P_{n}, n \in \mathbb{N}^{*}$. Step by step we follow the Gram-Schmidt procedure. Let us use $\langle f, g\rangle=\int_{0}^{y(0)} f(s) g(s) \mathrm{d} s$ as a dot product and $\|f\|^{2}=\langle f, f\rangle$ as a norm.

First let us define $\tilde{P}_{1}=P_{1} /\left\|P_{1}\right\|$ and $\pi_{1}=\left\langle\psi, \tilde{P}_{1}\right\rangle$ the projection of $\psi$ onto $\tilde{P}_{1}$. It is easy to check that $\pi_{1} \in \mathbb{R}$ depends only on $\dot{y}(0)$. We shall note $\left\langle\psi, \tilde{P}_{1}\right\rangle=\pi_{1}(\dot{y}(0))$.

Then, following the orthonormalization procedure, we define $\tilde{P}_{2}=g_{2} /\left\|g_{2}\right\|$ where $g_{2}=P_{2}-\left\langle P_{2}, \tilde{P}_{1}\right\rangle \tilde{P}_{1}$. We want to project $\psi$ onto $\tilde{P}_{2}$. Before doing so, let us consider the following result: for $p>1$ the coefficients $a_{2 p-1}$ and $a_{2 p}$ are of the polynomial form

$$
\begin{aligned}
a_{2 p-1} & =-y^{(p)}+h_{2 p-1}\left(\dot{y}, \ldots, y^{(p-1)}\right) \\
a_{2 p} & =-y^{(p)}(p \nu+(3-p) \dot{y})+h_{2 p}\left(\dot{y}, \ldots, y^{(p-1)}\right)
\end{aligned}
$$


where $h_{2 p-1}$ and $h_{2 p}$ are polynomials of their variables. This result is complemented by the special cases $a_{1}=-\dot{y}$ and $a_{2}=-\dot{y}(\nu+\dot{y})$. The proof follows from an easy induction. For sake of numerical experiments, exact expressions of the first five $a_{i}$ are given in the Appendix.

This last property allows us to state that the coefficients of the $P_{n}$ polynomial depend upon $\dot{y}(0), \ldots$, $y^{(n)}(0)$ only. Furthermore, by the orthonormal construction of the $\tilde{P}_{n}$, the coefficients of the $\tilde{P}_{n}$ depend upon $\dot{y}(0), \ldots, y^{(n)}(0)$ only. Thus the projection $\pi_{n}=\left\langle\psi, \tilde{P}_{n}\right\rangle$ depend only upon $\dot{y}(0), \ldots, y^{(n)}(0)$, and so

$$
\pi_{n}=\left\langle\psi, \tilde{P}_{n}\right\rangle=\pi_{n}\left(\dot{y}(0), \ldots, y^{(n)}(0)\right) .
$$

So far we have a projection of $\psi$ onto an orthonormal basis. We have now to recombine the obtained coefficients to derive conditions upon the $\left(a_{i}\right)$ coefficients. From

$$
\psi(x)=\sum_{i=1}^{\infty} \pi_{i}\left(\dot{y}(0), \ldots, y^{(i)}(0)\right) \tilde{P}_{i}
$$

and

$$
\tilde{P}_{i}=\sum_{j=i}^{\infty}\left\langle\tilde{P}_{i}, P_{j}\right\rangle P_{j}
$$

we get after recombination

$$
\psi(x)=\sum_{j=1}^{\infty} \sum_{i=1}^{j} \pi_{i}\left(\dot{y}(0), \ldots, y^{(i)}(0)\right)\left\langle\tilde{P}_{i}, P_{j}\right\rangle P_{j} .
$$

So it is possible to identify the coefficients in (18) for $j=1,2, \ldots$ as

$$
a_{2 j-1}=\sum_{i=1}^{j} \pi_{i}\left(\dot{y}(0), \ldots, y^{(i)}(0)\right)\left\langle\tilde{P}_{i}, P_{j}\right\rangle .
$$

Finally we substitute the expressions (19) and (20) in these last relations to get a set of equations to be solved in terms of the successive derivatives of $y$ :

$$
\left.\begin{array}{rl}
-\dot{y}(0) & =\pi_{1}(\dot{y}(0))\left\langle\tilde{P}_{1}, P_{1}\right\rangle \\
-y^{(2)}(0)+h_{3}(\dot{y}(0)) & =\pi_{1}(\dot{y}(0))\left\langle\tilde{P}_{1}, P_{2}\right\rangle+\pi_{2}(\dot{y}(0), \ddot{y}(0))\left\langle\tilde{P}_{2}, P_{2}\right\rangle \\
\vdots &
\end{array}\right\} .
$$

These equations are solvable for "small" initial conditions $x \mapsto \psi(x)$ in the $L^{2}$ sense. Indeed, the projection of such initial conditions onto the $\tilde{P}_{i}$ orthonormal polynomials are small, so the $\pi_{i}, i \in \mathbb{N}^{*}$ are small. This last property makes the set of equations solvable, since its jacobian gets closer to (minus) identity.

This procedure must be looked at in greater detail prior to any implementation. Density of the $\tilde{P}_{i}$ polynomials obtained through the Gram Schmidt procedure is an open issue (unfortunately the use of Stone-Weierstrass theorem is not straightforward and a dedicated approach seems required). Nonetheless numerical evaluations of the successive derivatives of $y$ corresponding to a prescribed initial condition seem tractable. While the number of such coefficients to be computed to approximate $\psi$ within some given tolerance is not known, we feel that such a result would be important for stabilization purposes. 


\section{Appendix A. Technical lemmas}

\section{Lemma A.1.}

$$
\frac{i ! j !(i+j+l+1) !}{(i+j+1) !}=\sum_{r=0}^{l}\left(\begin{array}{l}
l \\
r
\end{array}\right)(j+r) !(i+l-r) !, \quad i, \quad j, l \geq 0 .
$$

Proof. This result directly follows from the $\mathrm{Chu-Vandermonde} \mathrm{identity} \mathrm{[13]} \mathrm{that} \mathrm{gives}$

$$
(i+j+2)_{l}=\sum_{r=0}^{l}\left(\begin{array}{l}
l \\
r
\end{array}\right)(j+1)_{r}(i+1)_{l-r}
$$

where $(a)_{n}=a(a+1) \ldots(a+n-1)$ is the Pochhammer Symbol. One can use

$$
\begin{aligned}
(i+j+2)_{l} & =\frac{(i+j+l+1) !}{(i+j+1) !} \\
(j+1)_{r} & =\frac{(j+r) !}{j !} \\
(i+1)_{l-r} & =\frac{(i+l-r) !}{i !}
\end{aligned}
$$

and get after substitution

$$
\frac{i ! j !(i+j+l+1) !}{(i+j+1) !}=\sum_{r=0}^{l}\left(\begin{array}{l}
l \\
r
\end{array}\right)(j+r) !(i+l-r) !
$$

Lemma A.2. For $\alpha, c_{k} \geq 1$ and $b_{k} \geq 0, k=0,1, \ldots, l$,

$$
\sum_{k=0}^{l} c_{k}\left(b_{k}\right)^{\alpha} \leq\left(\sum_{k=0}^{l} c_{k} b_{k}\right)^{\alpha}, \quad l \geq 0
$$

The proof is an easy extension of that for the case $c_{k}=1, \forall k=0,1, \ldots, l$, given in [6].

\section{Appendix B. Gevrey functions Bounds}

In this section we give the derivation of the constants $M_{\phi}$ and $R_{\phi}$. The Gevrey function $\dot{\phi}$ used in the simulations is given by the function $\phi$, defined as

$$
\phi(\tau)= \begin{cases}L+\Delta L & \text { if } \tau \geq 1 \\ L+\Delta L g(\tau) & \text { if } 1>\tau>0 \\ L & \text { if } \tau \leq 0\end{cases}
$$

where,

$$
g(\tau)=\frac{f(\tau)}{f(\tau)+f(1-\tau)}, \tau \in[0,1] \quad \text { and } \quad f(\tau)= \begin{cases}\mathrm{e}^{-\frac{1}{\tau}} & \text { if } \tau>0 \\ 0 & \text { if } \tau \leq 0\end{cases}
$$


The function $\phi$ defines a smooth transition from $L$ to $L+\Delta L$ in liquid column length. The function chosen above is based upon an unpublished work of François Malrait done at École des Mines, which guarantees that

$$
g(\tau) \leq \frac{f(\tau)}{m} \text { where } 0<m \leq f(\tau)+f(1-\tau) .
$$

Given $f$ defined above, it is easy to show that $m=2 \mathrm{e}^{-2}$. The function $\dot{g}(\tau)$ is symmetric $(\dot{g}(\tau)=\dot{g}(1-\tau))$ so in estimating Gevrey bounds we can restrict the domain of $\tau$ to $[0,1 / 2]$. Also, $\mathbb{C} \ni z \mapsto g(z)$ is holomorphic in the infinite strip $\{z=x+i y \in \mathbb{C}: 0<x<1\}$. To identify estimates for the bounds on the Gevrey constants we utilize Cauchy's integral formula, namely for $g(\tau)$ we have

$$
g^{(k)}(\tau)=\frac{k !}{2 \pi r^{k}} \int_{-\pi}^{+\pi} \mathrm{e}^{-i k \theta} g\left(\tau+r \mathrm{e}^{i \theta}\right) \mathrm{d} \theta
$$

where $r \in] 0,1 / 2[$ and $k=1,2,3, \ldots$

Taking the absolute value we have

$$
\left|g^{(k)}(\tau)\right| \leq \frac{k !}{2 \pi r^{k}} \int_{-\pi}^{+\pi}\left|g\left(\tau+r \mathrm{e}^{i \theta}\right)\right| \mathrm{d} \theta \leq \frac{k ! \mathrm{e}^{2}}{4 \pi r^{k}} \int_{-\pi}^{+\pi}\left|f\left(\tau+r \mathrm{e}^{i \theta}\right)\right| \mathrm{d} \theta .
$$

Bounding the integrand

$$
\left|\mathrm{e}^{-1 /\left\{\tau+r \mathrm{e}^{i \theta}\right\}}\right|=\exp \left[\frac{-(\tau+r \cos (\theta))}{\tau^{2}+2 r \tau \cos (\theta)+r^{2}}\right] .
$$

Choosing $r=\mu \tau, 0<\mu<1$, we can simplify as

$$
\exp \left[\frac{-(\tau+r \cos (\theta))}{\tau^{2}+2 r \tau \cos (\theta)+r^{2}}\right]=\exp \left[\frac{-(1+\mu \cos (\theta))}{\tau\left(1+2 \mu \cos (\theta)+\mu^{2}\right)}\right] .
$$

The last expression in the brackets is an even function of $\theta \in[-\pi,+\pi]$ so we need only consider the behavior for $\theta \in[0,+\pi]$. Over this range, the expression is a decreasing function of $\theta$; to verify this, the derivative with respect to $\theta$ yields $-\left(\mu-\mu^{3}\right) \sin (\theta) / \tau$, which is always negative since $1>\mu>0$. Thus we can maximize the bracketed expression by evaluating it at $\theta=0$ as

$$
\exp \left[\frac{-(1+\mu \cos (\theta))}{\tau\left(1+2 \mu \cos (\theta)+\mu^{2}\right)}\right] \leq \exp \left[\frac{-(1+\mu)}{\tau(1+\mu)^{2}}\right]=\exp \left[\frac{-1}{\tau(1+\mu)}\right] .
$$

Returning to the integral equation with the definition for $r$ gives

$$
\frac{k ! \mathrm{e}^{2}}{4 \pi \mu^{k} \tau^{k}} \int_{-\pi}^{+\pi} \exp \left[\frac{-1}{\tau(1+\mu)}\right] \mathrm{d} \theta=\frac{k ! \mathrm{e}^{2}}{2 \mu^{k} \tau^{k}} \exp \left[\frac{-1}{\tau(1+\mu)}\right] .
$$

It is easy to show that

$$
\max _{\tau \in[0,1 / 2]}\left\{\tau^{-k} \exp \left[\frac{-1}{\tau(1+\mu)}\right]\right\} \leq \mathrm{e}^{-k} k^{k}(1+\mu)^{k}
$$

The bound now becomes

$$
\left.\frac{k ! \mathrm{e}^{2}}{2 \mu^{k} \tau^{k}} \exp \left[\frac{-1}{\tau(1+\mu)}\right] \leq \frac{k ! \mathrm{e}^{2}}{2}\left(\frac{k}{e}\right)^{k}\left(\frac{1+\mu}{\mu}\right)^{k}, \quad \text { for any } \mu \in\right] 0,1[.
$$


The best bound we can achieve is derived from the limiting behavior as $\mu \rightarrow 1$, resulting in

$$
\left|g^{(k)}(\tau)\right| \leq \frac{k ! \mathrm{e}^{2}}{2}\left(\frac{2 k}{e}\right)^{k} \simeq k !^{2} \frac{\mathrm{e}^{2}}{2 \sqrt{2 \pi}} \frac{2^{k}}{\sqrt{k}}, \quad \forall k=1,2,3, \ldots, \tau \in[0,1],
$$

where the last step utilizes Stirling's approximation. For reference, we also have the bounds

$$
\left|f^{(k)}(\tau)\right| \leq \frac{k !^{2}}{\sqrt{2 \pi}} \frac{2^{k}}{\sqrt{k}}, \quad \forall k=1,2,3, \ldots
$$

These parameterizations verify that $g$ and $f$ are Gevrey order 1 . As we want the Gevrey constants for $\dot{\phi}$, we can write the bounds as

$$
\left|\phi^{(k+1)}(\tau)\right|=\Delta L\left|g^{(k+1)}(\tau)\right| \leq \Delta L \frac{(k+1) !^{2} \mathrm{e}^{2} 2^{k+1}}{2 \sqrt{2 \pi(k+1)}}=\frac{\Delta L \mathrm{e}^{2}}{\sqrt{2 \pi}} k !^{2} 2^{k}(k+1)^{3 / 2}
$$

where $k=0,1,2, \ldots$ With the bounds $2^{k}(k+1)^{3 / 2} \leq 2^{k}(k+1)^{2} \leq 4^{k}$, we have bounds for $\dot{\phi}$ Gevrey order 1 , namely

$$
\left|\phi^{(k+1)}(\tau)\right| \leq M_{\phi} \frac{k !^{2}}{R_{\phi}^{k}}, \quad M_{\phi} \equiv \frac{\Delta L \mathrm{e}^{2}}{\sqrt{2 \pi}}, R_{\phi} \equiv \frac{1}{4}
$$

The value of the column length increase $\Delta L$ is the only non-constant in these Gevrey bounds. To characterize these bounds, consider normalizing the model coefficients $\rho=\nu=1$ and examine the radius of convergence $\eta^{*}$ as a function of column length as shown in Figure 9, where we assume $0<L \ll 1$. For guaranteed convergence of the solution over the domain, $\eta^{*}>\Delta L$ and the plot shows that this is the case when $\Delta L \in[0.0,0.45]$. For an initial column length $L>0.6$, a time bound $T$ larger than 1 is also clearly required for even small column length increases.

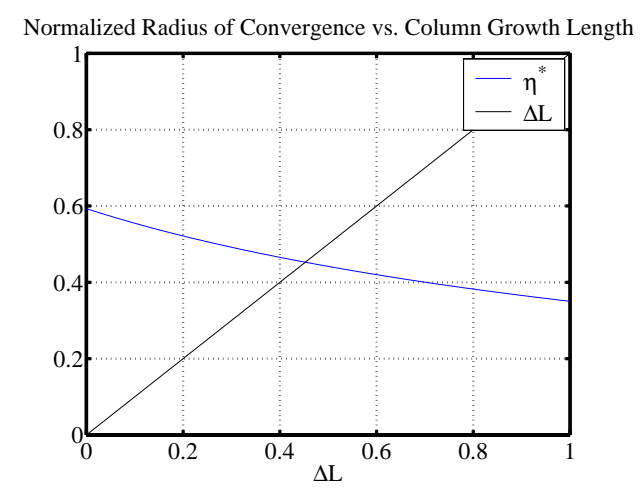

FIGURE 9. For normalized model coefficients $(\rho=\nu=1)$, the locus of the radius of convergence $\eta^{*}$ as a function of the column length increase $\Delta L$.

\section{REFERENCES}

[1] J.R. Cannon, The one-dimensional heat equation. Addison-Wesley Publishing Company, Encyclopedia Math. Appl. 23 (1984).

[2] M. Fila and P. Souplet, Existence of global solutions with slow decay and unbounded free boundary for a superlinear Stefan problem. Interfaces Free Boundaries 3 (2001) 337-344.

[3] M. Fliess, J. Lévine, Ph. Martin and P. Rouchon, Flatness and defect of nonlinear systems: Introductory theory and examples. Int. J. Control 61 (1995) 1327-1361. 
[4] M. Fliess, J. Lévine, Ph. Martin and P. Rouchon, A Lie-Bäcklund approach to equivalence and flatness of nonlinear systems. IEEE Trans. Automat. Control 44 (1999) 922-937.

[5] A. Friedman and B. Hu, A Stefan problem for multidimensional reaction-diffusion systems. SIAM J. Math. Anal. 27 (1996) $1212-1234$.

[6] M. Gevrey, La nature analytique des solutions des équations aux dérivées partielles. Ann. Sci. École Norm. Sup. 25 (1918) 129-190.

[7] C.D. Hill, Parabolic equations in one space variable and the non-characteristic Cauchy problem. Comm. Pure Appl. Math. 20 (1967) 619-633.

[8] Chen Hua and L. Rodino, General theory of partial differential equations and microlocal analysis, in Proc. of the workshop on General theory of PDEs and Microlocal Analysis, International Centre for Theoretical Physics, Trieste, edited by Qi Min-You and L. Rodino. Longman (1995) 6-81.

[9] B. Laroche, Ph. Martin and P. Rouchon, Motion planing for the heat equation. Int. J. Robust Nonlinear Control 10 (2000) 629-643.

[10] A.F. Lynch and J. Rudolph, Flatness-based boundary control of a nonlinear parabolic equation modelling a tubular reactor, edited by A. Isidori, F. Lamnabhi-Lagarrigue and W. Respondek. Springer, Lecture Notes in Control Inform. Sci. 259: Nonlinear Control in the Year 2000, Vol. 2. Springer (2000) 45-54.

[11] M.B. Milam, K. Mushambi and R.M. Murray, A new computational approach to real-time trajectory generation for constrained mechanical systems, in IEEE Conference on Decision and Control (2000).

[12] N. Petit, M.B. Milam and R.M. Murray, A new computational method for optimal control of a class of constrained systems governed by partial differential equations, in Proc. of the 15th IFAC World Congress (2002).

[13] M. Petkovsek, H.S. Wilf and D. Zeilberger, $A=B$. Wellesley (1996).

[14] L.I. Rubinstein, The Stefan problem. AMS, Providence, Rhode Island, Transl. Math. Monogr. 27 (1971).

[15] W. Rudin, Real and Complex Analysis. McGraw-Hill International Editions, Third Edition (1987). 